\title{
The Impact of Secondary Notation on Process Model Understanding
}

\author{
Matthias Schrepfer ${ }^{1}$, Johannes Wolf ${ }^{1}$, Jan Mendling ${ }^{1}$, and Hajo A. Reijers ${ }^{2}$ \\ 1 Humboldt-Universität zu Berlin \\ Unter den Linden 6, 10099 Berlin, Germany \\ jan.mendling@wiwi.hu-berlin.de \\ 2 Eindhoven University of Technology \\ PO Box 513, 5600 MB Eindhoven, The Netherlands \\ h.a.reijers@tue.nl
}

\begin{abstract}
Models of business processes are usually created and presented using some visual notation. In this way, one can express important activities, milestones, and actors of a process using interconnected graphical symbols. While it has been established for other types of models that their graphical layout is a factor in making sense of these, this aspect has not been investigated in the business process modeling area. This paper proposes a set of propositions about the effects of the secondary notation, which entails layout, on process model comprehension. While individual graphical readership and pattern recognition skills are known mediators in interpreting visual cues, these propositions take expertise into account. The goal of this paper is to lay the foundation of follow-up, empirical investigations to challenge these propositions.
\end{abstract}

Keywords: process modeling, secondary notation, comprehension, modeling expertise.

\section{Introduction}

Business process models have become an integral part of organizational engineering efforts. They are used both on the business level for describing business operations in a consistent way as well as on the technical level for specifying requirements that have to be supported by enterprise software. As a consequence, business process design is now one of the major reasons for conducting conceptual modeling projects 14. The process models created in these initiatives capture among others what tasks, events, states, and control flow logic constitute a business process. The different symbols for these elements are part of the graphical notation of process modeling languages. Using a process modeling tool, these symbols are typically placed on a modeling canvas and connected with arcs. The result is a visual model that represents a business process.

Business process models play an important role in facilitating documentation and communication between different stakeholders in a process design project. 
Therefore, they should be created in a way to best serve this purpose. A major requirement is that they reveal their content in an intuitive and easily understandable manner [15]. Prior research has shown that several factors influence the understanding of a process model; for instance, complex models created by human modelers are more likely to contain errors 26, modeling expertise appears to improve understanding performance 24], and characteristics of the modeling notation have a direct impact on comprehension [19].

While these factors are well covered by current research, the influence of graphical layout on understanding is partly acknowledged (e.g. in [24]) but not yet thoroughly investigated for process models. We address this research gap by revisiting empirical findings on graph aesthetics in this paper. We also discuss in detail how modeling expertise interacts with the quality of the graphical layout of a process model. Our contribution is a set of propositions that builds on a sound theoretical foundation of cognitive research. These propositions are a starting point for investigating the influence of graph layout on process model understanding empirically.

The paper proceeds accordingly. Section 2 motivates the importance of graphical layout by the help of a process model example. Furthermore, we introduce secondary notation as a cognitive dimension relevant to this problem. Section 3 discusses expertise as an important factor that interacts with layout quality. We revisit related work on computer programme comprehension, and discuss its relevance to process model layout. Section 4 presents propositions that build on the theoretical discussion of the previous sections. In Section 5 we discuss different measurement options for an operationalization of the propositions in an experiment. Section 6 closes the paper with a conclusion and an outlook on future research.

\section{Graphical Layout and Understanding}

This section discusses the relevance of graphical layout for model understanding. We stick to the Business Process Modeling Notation (BPMN) to illustrate our argument. The BPMN specification provides a standardized graphical notation that is meant to be easily understandable by all relevant stakeholders [37. It offers notation elements for activities, events, and routing conditions (gateways), which are connected by control flow arcs.

Figure 1 presents a sales process modeled in BPMN. The process starts with the submission of a quote. The customer and the company then negotiate the contract. If both parties cannot agree on the contract, they can re-negotiate it. Instead, they can also decline the contract such that it is archived. Once the parties agree on the contract, the responsible department approves it depending on the contract value. If it is below five million dollar, the sales department approves the contract. If the value is larger or equal to that amount, the regional manager must approve the contract. Following the approval, the user deal is concluded. After the contract is archived, the business process ends.

The whole process in Figure 1 is built using different BPMN notation elements that are connected by arcs. The process is initiated with a start event which is 


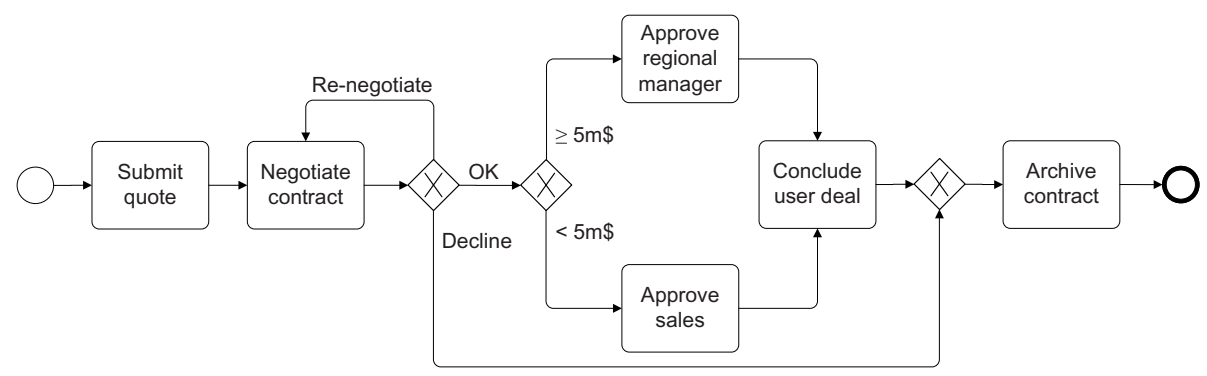

Fig. 1. Sales Process Model in BPMN (Good Layout)

depicted as a circle. There are different events in BPMN which usually affect the process flow. Events occur due to a trigger or they mark a result [37. Roundedcorner rectangles mark activities in the process model, which represent different tasks to be performed within a process. After the activity 'Negotiate Contract' an XOR gateway is reached and shown as a diamond shape. It defines a decision point. BPMN offers different gateways including XOR, AND, and OR to control the sequence flow in a business process model. The BPMN process terminates with an end event, drawn as a circle with a bold line.

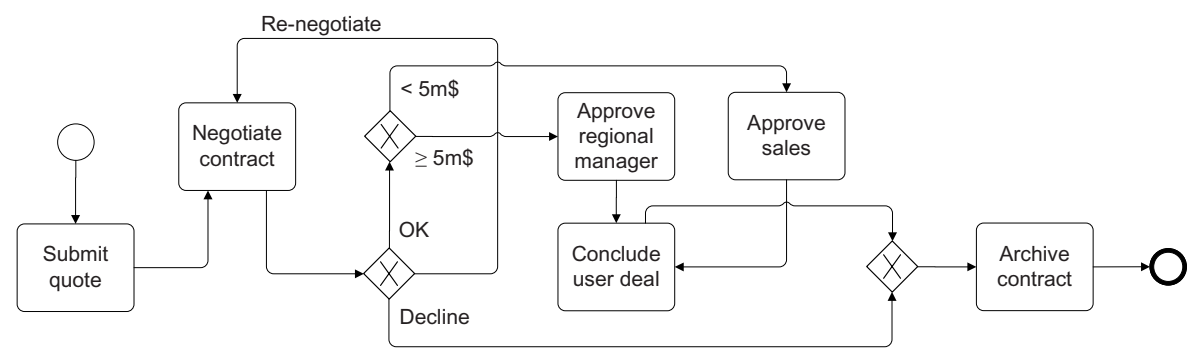

Fig. 2. Sales Process Model in BPMN (Bad Layout)

Although BPMN specifies the set of notation elements to be used in a process model, it does not make any normative statements on how the elements should be positioned. Figure1 1 and Figure 2 illustrate this fact by showing the same sales process using two different layouts. Although the processes differ in their visual representation, the process models are identical from a logical point of view. Their semantics are the same, but their effect on the human reader might still be different in terms of understanding. Cognitive research into program comprehension has coined the terms first notation and secondary notation to describe this phenomenon. The modeling notation as a formal set of symbols is defined as first notation. First notation specifies the semantics of all graphical elements of 
a particular notation such as BPMN. However, the visual characteristics of the model are not limited by first notation. By enriching the process model with information beyond the formal notation (e.g. color, line strength, etc.), the reader may access the information captured in the model with a differing degree of ease 28. Visual cues, which are not part of a notation, are known as secondary notation 2829 . Among others, the model designer is free in selecting the graphical position of model elements. Accordingly, we can state that the models presented in Figure 1 and Figure 2 differ in terms of secondary notation. Although these visual cues do not change the semantics of a model, they have been identified as an important factor of model comprehension in prior research [27/28].

It is important to note that secondary notation is not constrained in its use. Graphical layout is one particular aspect of secondary notation that is known to influence understanding [30. It is an appealing feature of graphical layout that it can be traced back to a number of layout parameters. For some domains, e.g. electronics, several hints and rules exist how and when secondary notation should be used [20. Also model designers often use rules of thumb when changing layout parameters 28. The priority of these rules may even be adjusted while a model is created. Prior research has shown that the comprehension of graphical layout of a model depends upon a number of parameters 1028 30 31. Some of these parameters have been identified as having a strong influence with respect to the understanding of a model 28:30. In the following, the most relevant layout factors with respect to graphical layout are presented by mentioning their effect on overall understanding and by relating them to the two versions of the sales process.

- Line Crossings: The higher the number of crossings within a graphical layout, the lower the readability of that layout 28]. In 30] it is shown that line crossings influence the understanding most of all. The model in Figure 1 does not contain any line crossings while the model in Figure 2 contains three crossings.

- Edge Bends: The number of edge bends negatively affects the understanding of a process model [30 31]. The graphical layout of the process model in Figure 1 contains eight edge bends, the model shown in Figure 2 exhibits 12 bends.

- Symmetry: Graphical layouts where elements are placed more symmetric are easier readable. However, the effect of symmetry is lower than the one of line crossings 3031. The graphical elements, activities 'Approve regional manager' and 'Approve sales', are shown symmetrically in the model presented in Figure 1] while in the other model they are not placed symmetrically.

- Use of Locality: Graphical elements which are related to each other shall be placed close to each other making them easier recognizable and leading to a higher understanding of the model [29]. The activity 'Approve sales' in the model depicted in Figure 2 is not positioned close to the preceding XOR-gateway. 
The significance of these layout parameters for comprehension builds on cognitive insights regarding the process of reading and understanding a process model. There are two aspects of this process that are influenced by the visual representation. Graphical readership describes the ability to read a business process model. This means, first, to identify the graphical elements and visual cues shown in the model, and second, to interpret their individual meaning specified in the modeling notation 1328 . The process of reading a process model is less a matter of intuition because the reader has to understand the semantics of modeling notation. Order is an important concept of process models, and Figure 2 aims to illustrate that it can be obfuscated by unappropriate layout. The second important aspect is pattern recognition. As the term emphasizes, a model usually contains certain patterns that altogether describe a specific behavior within a business process. Several workflow patterns such as the Exclusive Choice have been described in [1]. A model reader must recognize a pattern in the graphical layout in order to access the information described by the pattern. The sales process includes the Exclusive Choice pattern combined with a Simple Merge. Together they define a more complex decision block, which is obfuscated in Figure 2] Furthermore, there is a Structured Loop pattern (see 34]) at the activity 'Negotiate contract'. Again, this pattern is easily visible in Figure 1, but not in Figure 2. In large process models, it is likely to be more difficult to recognize workflow patterns that are obfuscated by bad layout. Both aspects, graphical readership and pattern recognition, are influenced by layout parameters, and they contribute to the understanding of a process model.

\section{$3 \quad$ Modeling Expertise and Understanding}

In the previous section we identified the impact of layout on graphical readership and pattern recognition. Clearly, graphical readership and pattern recognition skills vary between readers of a model. Therefore, model comprehension has to be correlated with these individual skills.

Consider again the two versions of BPMN sales model from the previous chapter, and assume it is presented to both a first-year Bachelor's student in Information Systems, and a professor with a research focus on process modeling. Both individuals get the task to read and interpret these process models. Even without a proper analysis of knowledge and skills of both individuals, we would assume that the professor is much faster and more accurate in understanding compared to the first-year student. The advantage of the professor can be traced back to his gained expertise and his extensive knowledge in the field of process modeling. This does not only cover the graphical elements of the notation (graphical readership) which might be new or even unfamiliar to the student. Furthermore, the professor will likely be able to recognize common patterns in the model (pattern recognition). Besides these rather obvious statements, the comparison between the student and the professor is not directly clear when the layout is changed. How will the variation in layout influence the relative performance? Before giving a preliminary answer to this question, we revisit different aspects and constructs related to expertise. 
The importance of modeling expertise for process modeling has been confirmed in different works. The survey by Bandara on success factors of process modeling establishes modeler expertise as a critical issue [6]. This success factor describes "the experiences of the person conducting the modeling, in terms of conceptual modeling in general and process modeling in particular." A set of sub-constructs is also identified including required skills, knowledge and experience. In this way, the factor also covers the criterion user training that captures the extent of knowledge given to a users about the modeling tool and modeling procedures. Language expertise is mentioned in [5] as a more specific factor. Corresponding scales have been defined by Recker for familiarity with a modeling language.

Expertise as a general factor for process modeling comprehension has also been confirmed in experimental designs. Mendling and Strembeck find that theoretical knowledge has a significant influence on process model understanding such that experienced modelers perform better in understanding [25]. Similar observations are made in [24] where understanding tasks were presented to 73 students from three European universities. A significant difference was observed in comprehension performance among the three group, which was traced back to a broader and deeper teaching of Petri nets at one university. This study discusses a potential threat to validity in terms of expertise. Student could be classified as novices, such that the results would not hold for professionals who are experts in the field. For this reason, the results are discussed with 12 professional process modelers for their conclusiveness [24]. In an experiment on modularity, 28 Dutch process consultants are involved who are assumed to be experts 33 .

The definition of the concept of expertise turns out to be quite challenging. In demotic definitions the condition of being an expert is used to describe the term expertise. It is often related to extensive skill or knowledge in a particular field 36]. Green et al. describe expertise from a cognitive sciences point of view. "Expertise is not only a characteristic of higher-order cognitive logic but also of perceptual logic, that can be trained to better support cognitive operations through "perceptual expertise" [18. The aspect that expertise is based on trained skills and gained knowledge seems to be of the utmost importance. The field of psychology has analyzed the notion of expert and exceptional performance [16]. There, expert performance is characterized by a "varying balance between training and experience (nurture) on one hand and innate differences in capacities and talents (nature) on the other" whereas "experts knowledge and task-specific reactions must have been acquired through experience". This understanding builds upon the theory of expertise formulated by Chase and Simon in 1973 [12. They postulated that expertise is "the result of acquiring, during many years of experience in their domain, vast amounts of knowledge and the ability to perform pattern-based retrieval". Although expertise is often equated with "the amount and complexity of knowledge gained through extensive experience of activities in a domain", it is stated that this criterion is not sufficient for measuring an individual's performance on a task [16]. Bonner and Pennington 
noticed that "most experts are highly specialized, and task-specific experience is a better, but still modest, predictor of performance" [8].

An important feature of expertise can be highlighted by the so-called 10-years rule of necessary preparation. According to this rule, not even the most talented individuals can attain international performance without approximately 10 years of preparation. Ericsson and Lehmann found out that the mere number of years of experience with relevant activities in a domain is typically only weakly related to performance [16]. Individualized training activities designed to improve specific aspects of an individuals performance through repetition and successive refinement seem to play a more important role because they cause physiological and neurological adaption in the body. These training activities where labeled by Ericsson and Lehmann as deliberate practice. They found out that a daily amount of four hours of fully concentrated training appears to be a sustainable basis for most humans. In addition, the correlation of talent and expert performance suggests that talent, i.e. innate domain-specific basic capacities, has a small, possibly negligible impact on expert performance. Hence, an individual's performance who solely gained years of expertise is much lower than those of an expert who took part in an substantial amount of training and practice. Experts make extensive use of planning, reasoning, anticipating and controlling in order to face new learning tasks and to increasingly improve their performance.

There are some perspectives on expertise that are prone to wrong conclusions. The levels of expertise are often discussed by referring to the terms novice and expert in order to indicate a rather weak or strong expertise (see e.g. the notion of 'expert modeler' in [5]). This distinction is problematic because there exists no clear definition of both terms, making it difficult to unambiguously identify people as novices or experts. In this way, the spectrum between low and high expertise is ignored. The terms novice and expert are sometimes also used in a simplifying way by equating novices with students and experts with professionals, which is an classification to be justified. Beyond that, relying on a self-assessment has been found unsuitable for identifying novices and experts in different works. In 24 it was shown that self-assessment of students concerning their process modeling knowledge is not correlated to their performance in understanding. Similar observations have been made in 9]. This clarifies the necessity of an objective and systematic assessment of a person's process modeling expertise.

Summing up, current research makes wide use of the terms novice and expert but evades to define these constructs and tends to use them in a demotic way. This approach may be sufficiently for nominally distinguishing between weak and strong expertise, but can hardly be used for more finely granulated levels of distinction. When talking about process model understanding based on a model's secondary notation, a more detailed distinction between levels of expertise would be useful. Therefore a combination of the presented approaches should be considered using a combination of information given by the individual itself (e.g. duration and frequency of practical experience and training), plus additionally an objective measure of the individual's skills (e.g. the ability of 
Table 1. The different Aspects of Expertise

\begin{tabular}{l|l}
\hline Aspect & Authors \\
\hline $\begin{array}{l}\text { Modeler expertise (skills, knowledge, experience) } \\
\text { User training and competence }\end{array}$ & Bandara [6] p.169] \\
\hline Modeling language expertise & Aranda []] \\
\hline Familiarity with language & Recker [32] \\
\hline $\begin{array}{l}\text { Self-assessment of theoretical knowledge } \\
\text { Self-assessment of practical experience }\end{array}$ & Mendling, Reijers, Cardoso [24] \\
Students versus Practitioners & \\
\hline Theoretical knowledge & Mendling and Strembeck [25] \\
Time of being involved with process modeling \\
Intensity of being involved with process modeling
\end{tabular}

pattern-recognition and graphical readership). In contrast to earlier research experiments, this approach should highlight the aspect of individual skills and abilities and do not only focus on the amount of both theoretical and practical knowledge and experience. An important step will be to reveal how and to what extent individuals gained perceptual expertise through intensive training and make use of certain mechanisms, e.g. planning, reasoning, anticipating and controlling, that distinguishes the expert from the novice. Table 1 lists the different aspects of expertise, that piece together the term expertise.

\section{Propositions}

In this section, we bring together observations on layout and expertise as factors of process model understanding. We argue that the effect of secondary notation on model comprehension must also take individual expertise into account, as recognition capabilities can be trained. Indeed, the interaction between a model's 


\section{Model Understanding}

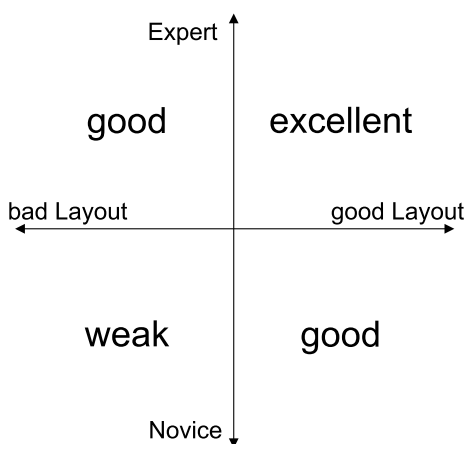

Fig. 3. 2-dimensional 2x2 Matrix displaying Process Model Understanding

layout quality and modeling expertise is partly considered in several studies. One of the most comprehensive studies concerning the layout-expertise interaction was undertaken by Petre 28. When analyzing readership skills and graphical programming, Petre discovered that novice modelers usually create models that are less comprehensible due to poor use of the secondary notation. The secondary notation allows expert modelers to concentrate on relevant graphical elements, to disregard irrelevant information and recognize patterns. Furthermore, novice users of graphics tend to lack reading and search strategies. These strategies correlate with modeling experience and are the result of extensive learning.

Building on the arguments of the previous sections, we can assume that:

- Given an arbitrary process model, a good graphical layout increases model understanding, whereas a bad graphical layout decreases model understanding.

- Given an arbitrary process model, an expert performs significantly better when it comes to model understanding, compared to an individual classified as a novice.

Based on these two basic propositions, we can reason about the joint impact of graphical layout and expertise on process model understanding. Let us again consider the professor and the student trying to comprehend a process model. We illustrate the problem in a 2-dimensional matrix, including graphical layout and expertise as the two axis (see Figure 3). This matrix contains four areas for each combination of the two factors. According to propositions (1) and (2), the professor would perform significantly better than the student on both good and bad layout. Furthermore, good layout would be understood significantly better by both professor and student. We expect that the student will struggle with the bad layout, whereas he is able to cope with the good one. Instead, the professor is able to perform well even with the bad layout, whereas he performs excellent with the good one.

Taking the consolidated findings from the previous sections into account, we are able to formulate the following propositions: 
H1a. For process models with bad layout, experts are likely to perform significantly better than novices for tasks of model comprehension. We expect that experts, although layout cues are obfuscated, can demonstrate their knowledge of reading and understanding information from the models. Experts are presumably capable to recognize hidden workflow patterns and visual cues using their modeling expertise and perceptual training. Contrarily, we expect novices to lack practical expertise on graphical readership and pattern recognition. Therefore, novices face problems of perceiving and interpreting relevant information in process models.

H1b. For process models with layout that use secondary notation for providing visual cues, we expect that experts perform significantly better than novices for comprehension tasks. We assume that experts exhibit their perceptual and practical expertise especially when good layouts are presented. Due to their gained experience experts perform faster and better than novices in understanding these process models. Novices might be slower as they lack efficient reading and search techniques in order to access information in a fast way.

H2a. For novices with a lack of modeling expertise, we hypothesize that good layout of process models significantly enhances model understanding. Bad layout makes it difficult or impossible for novices to extract information while good layout greatly supports the information extraction process. Novices might not recognize workflow patterns in badly layouted models due to missing expertise. Thus, they cannot uncover all information shown in the layout. Therefore, process models with a good layout support the understanding of novices.

H2b. For experts with practical and theoretical experience, we postulate that bad layout of process models significantly decreases the performance of model understanding. We expect experts being able to extract and interpret information from bad layouts with the help of perceptual expertise. However, good layout should increase the performance of experts as it supports graphical readership and pattern recognition. Even though experts know sophisticated search strategies, bad layouts hinders both abilities of graphical readership and pattern recognition. Therefore, we expect a higher understanding of experts for good layout.

H3. We expect that the effect of layout on model understanding will be greater for novices than for experts. Experts will be faster and more accurate in reading relevant information from a process model, even if it is obfuscated by bad layout. Since novices do not know appropriate search strategies, they will be more dependant on an intuitive presentation of the information using a supportive process model layout.

Altogether, we assume that both groups will be affected by the way how the process model is graphically laid out, but this impact will be greater for novices. 


\section{Experimental Setup}

In this section, we discuss the experimental setup. We will focus on potential interactions, and how we aim to track them. Section 5.1 elaborates on the stimulus and the general setup of the experiment. Section 5.2 discusses the dimensions of performance that we aim to measure. Section 5.3 emphasizes potential interaction effects, and how we aim to deal with them.

\subsection{Stimulus}

In order to investigate the impact of graphical layout on process model understanding, we have to use structurally equivalent models with different layout. There are different challenges in this regard. First, due to learning effects, it is not possible to show the same model to the same person, once with good and once with bad layout. We therefore have to use a block design where participants are randomly assigned to one of two groups such that they either see the good or the bad version of the model. Second, we have to investigate the level of variation in layout parameters in order to find out which changes are significant. The authors of [7] conducted a pre-test to study the strength of impact of their stimulus. Based on the results they used three classes of variation. Analogously, we plan to investigate how huge the variation in layout parameters like bending points and edge crossings should be in the experiment to have a potential impact.

\subsection{Comprehension Performance}

We aim to measure comprehension performance in different dimensions. Accuracy (number of correct answers), efficiency (comprehension time), and efficacy (accuracy divided by time) are the major performance measures in the context [17. Since layout is highly perceptual, we also aim to record perceived difficulty. The selection of questions on model comprehension requires specific care. It is common practice to utilize questions, for which an answer can be objectively judged to be correct or wrong [24 25. This call for objectivity implies ignoring the (informal) content of activity labels, and using abstract letters as activity names instead. The focus will then be on binary relationships between two activities in terms of execution order, exclusiveness, concurrency, and repetition. These relationships play an important role for reading, modifying, and validating the model. Respective statements such as "Executing activity $a_{i}$ implies that $a_{j}$ will be executed later" can be easily verified using the reachability graph of the process model. A reachability graph captures all states and transitions represented by the process model and it can be (automatically) generated from it. For some classes of models, several relationship can be calculated more efficiently without the reachability graph. For instance, the concurrency relation can be constructed for those process models that map to free-choice Petri nets in $O\left(n^{3}\right)$ time 22 .

There is also a debate on how representative a question is for the overall model understanding. As the number of questions can become quite large for 
estimating understanding within the boundaries of a given confidence interval [21, we rather plan to identify difficult questions based on a notion of distance in the process model graph. A different option is to choose questions randomly, and later calculate measures of consistency like Cronbach's Alpha for the actually given answers. The rationale here would be that consistent sets of answers were likely to be a good estimate for general model understanding. The disadvantage of the latter option is that confidence in the measurement can only be established post-hoc. We expect a greater variation in understanding by using difficult questions instead of selecting random questions. Still, it can easily be combined with a consistency analysis after gather data.

\subsection{Potential Interactions}

There are several factors that might have a potential influence on process model understanding. In the previous sections, we have already discussed that we aim to analyze different aspects of process modeling expertise as main covariates. There are other factors discussed in literature including model size and complexity, domain knowledge, modeling purpose, and modeling notation.

The importance of model characteristics is the foundation for work into process model metrics. Metrics have been defined for different structural and behavioral aspects of a process model including control-flow complexity (CFC) [1], size, complexity, and coupling [4, modularity [2, or structuredness 26]. Their impact on model understanding and error probability has been studied in different works - see 26] for an overview. In our experiment we aim to neutralize the impact of size and structure on understanding as much as possible. Similar to 24 we plan to choose models of comparable size.

Domain knowledge might also have an impact. If someone is knowledgable in health-care and he answers questions on hospital process models, it is not directly clear whether a good performance can be attributed to model comprehension or domain knowledge. In general, people may find it easier to read a model about the domain they are familiar with than other models. While this has not been established for process models, it is known from software engineering that domain knowledge affects the understanding of particular code [23. We aim to neutralize the impact of domain knowledge by using abstract letters as activity names.

The understanding of a model may be affected by the specific purpose the modeler had in mind. The best example is that some process models are not intended to be used on a day-to-day basis by people but instead are explicitly created for automatic enactment. In such a case, less care will be given to make them comprehensible to humans. The differences between process models as a result of different modeling purposes are mentioned, for example, in [15]. Empirical research into this factor is missing.

In the presence of many different notations for process models, e.g. UML Activity diagrams, EPCs, BPMN, YAWL, and Petri nets, it cannot be ruled out that some of these are inherently more suitable to convey meaning to people than others. Empirical research that has explored this difference is, for example, reported in 35]. According to these publications, the impact of the notation 
being used is not very high, maybe because the languages are too similar. Similar observations are made in 32. Other research that compares notations of a different focus identify a significant impact on understanding 193. We try to neutralize the impact of the notation on an experiment by using the BPMN notation. It is a widely accepted standard and it covers those routing elements that are also found in other languages like EPCs or YAWL. Furthermore, we will focus on those BPMN elements actually used in modeling practice 38.

\section{Conclusion}

This paper is motivated by the importance of comprehension for the use of business process models. We have discussed expertise and graphical layout as two important factors affecting the ease of process model comprehension. Our contribution is a set of hypotheses that build on a sound theoretical foundation. In particular, we argue that graphical layout influences model comprehension for both experts and novices, but that the effect would be greater for the latter. From these arguments, our plans for future work follow naturally: the proposed hypotheses will be challenged by empirical research that we plan to conduct at our universities in Berlin and Eindhoven.

A clear limitation of our work that needs to be noted is that further interacting factors may be at work that influence process model comprehension. For example, some notations display a wider variety of graphical constructs than others, so that the considered process modeling language may mediate the effect of the secondary notation as we discussed. Considering the early state of research in the area of process model understanding, we see no other option than to try and study the various factors in combinations that are manageable from an experimental perspective. At the same time, it would be a very welcome development if other researchers would engage in these topics, so that progress can be made in distinguishing the most important interactions. Indeed, the presentation of our hypotheses at this stage may be considered as an explicit encouragement and invitation.

\section{References}

1. van der Aalst, W.M.P., ter Hofstede, A.H.M., Kiepuszewski, B., Barros, A.P.: Workflow Patterns. Distributed and Parallel Databases 14(1), 5-51 (2003)

2. van der Aalst, W.M.P., Lassen, K.B.: Translating unstructured workflow processes to readable BPEL: Theory and implementation. Information and Software Technology 50(3), 131-159 (2008)

3. Agarwal, R., De, P., Sinha, A.P.: Comprehending object and process models: An empirical study. IEEE Transactions on Software Engineering 25(4), 541-556 (1999)

4. Rolón Aguilar, E., García, F., Ruiz, F., Piattini, M.: An exploratory experiment to validate measures for business process models. In: First International Conference on Research Challenges in Information Science, RCIS (2007) 
5. Aranda, J., Ernst, N., Horkoff, J., Easterbrook, S.: A framework for empirical evaluation of model comprehensibility. In: MISE 2007: Proceedings of the International Workshop on Modeling in Software Engineering, Washington, DC, USA, p. 7. IEEE Computer Society, Los Alamitos (2007)

6. Bandara, W., Gable, G.G., Rosemann, M.: Critical success factors of business process modeling (2007)

7. Batra, D., Wishart, N.A.: Comparing a rule-based approach with a pattern-based approach at different levels of complexity of conceptual data modelling tasks. Int. J. Hum.-Comput. Stud. 61(4), 397-419 (2004)

8. Bonner, S.E., Pennington, N.: Cognitive processes and knowledge as determinants of auditor expertise. Journal of Accounting Literature 10(1), 1-50 (1991)

9. Burton-Jones, A., Meso, P.: How good are these uml diagrams? an empirical test of the wand and weber good decomposition model. In: Applegate, L., Galliers, R., DeGross, J.I. (eds.) Proceedings of the Twenty-third International Conference on Information Systems (ICIS), pp. 101-114 (2002)

10. Byron, L., Wattenberg, M.: Stacked graphs - geometry \& aesthetics. IEEE Transactions on Visualization and Computer Graphics 14(6), 1245-1252 (2008)

11. Cardoso, J.: Evaluating Workflows and Web Process Complexity. In: Workflow Handbook 2005, pp. 284-290. Future Strategies, Inc. (2005)

12. Chase, W.G., Simon, H.A.: The mind's eye in chess. Visual Information Processing (1973)

13. Chattratichart, J., Kuljis, J.: Some Evidence for Graphical Readership, Paradigm Preference, and the Match-Mismatch Conjecture in Graphical Programs. In: 13th Workshop of the Psychology of Programming Interest Group (2001)

14. Davies, I., Green, P., Rosemann, M., Indulska, M., Gallo, S.: How do practitioners use conceptual modeling in practice? Data \& Knowledge Engineering 58(3), 358380 (2006)

15. Dehnert, J., van der Aalst, W.M.P.: Bridging The Gap Between Business Models And Workflow Specifications. International J. Cooperative Inf. Syst. 13(3), 289-332 (2004)

16. Ericsson, K.A., Lehmann, A.C.: Expert and exceptional performance: Evidence of maximal adaptation to task constraints. Annual Review of Psychology 47(1), 273-305 (1996)

17. Gilmore, D.J., Green, T.R.G.: Comprehension and recall of miniature programs. International Journal of Man-Machine Studies 21(1), 31-48 (1984)

18. Green, T.M., Ribarsky, W., Fisher, B.: Building and applying a human cognition model for visual analytics. Information Visualization 8(1), 1-13 (2009)

19. Hahn, J., Kim, J.: Why are some diagrams easier to work with? effects of diagrammatic representation on the cognitive integration process of systems analysis and design. ACM Transactions on Computer-Human Interaction 6 (1999)

20. Horowitz, P., Hill, W.: The art of electronics, 2nd edn. University Press, Cambridge (1989)

21. Reijers, H.A., Seese, D., Melcher, J., Mendling, J.: On measuring the understandability of process models. In: Proceedings of BPM Workshops 2009 - ER-BPM Workshop. Springer, Heidelberg (to appear)

22. Kovalyov, A., Esparza, J.: A polynomial algorithm to compute the concurrency relation of free-choice signal transition graphs. In: Prof. of the International Workshop on Discrete Event Systems, WODES 1996, Edinburgh, pp. 1-6 (1996)

23. Lakhotia, A.: Understanding someone else's code: Analysis of experiences. Journal of Systems and Software 23(3), 269-275 (1993) 
24. Mendling, J., Reijers, H.A., Cardoso, J.: What makes process models understandable? In: Alonso, G., Dadam, P., Rosemann, M. (eds.) BPM 2007. LNCS, vol. 4714, pp. 48-63. Springer, Heidelberg (2007)

25. Mendling, J., Strembeck, M.: Influence factors of understanding business process models. In: Abramowicz, W., Fensel, D. (eds.) BIS 2008. LNBIP, vol. 7, pp. 142153. Springer, Heidelberg (2008)

26. Mendling, J.: Metrics for Process Models: Empirical Foundations of Verification, Error Prediction, and Guidelines for Correctness. LNBIP, vol. 6. Springer, Heidelberg (2008)

27. Moher, T.G., Mak, D.C., Blumenthal, B., Leventhal, L.M.: Comparing the Comprehensibility of Textual and Graphical Programs: The Case of Petri Nets. In: Cook, C.R., Scholtz, J.C., Spohrer, J.C. (eds.) Empirical Studies of Programmers: Fifth Workshop: Papers Presented at the Fifth Workshop on Empirical Studies of Programmers, December 3-5, pp. 137-161. Ablex Pub. (1993)

28. Petre, M.: Why looking isn't always seeing: readership skills and graphical programming. Commun. ACM 38(6), 33-44 (1995)

29. Petre, M.: Cognitive dimensions 'beyond the notation'. J. Vis. Lang. Comput. 17(4), 292-301 (2006)

30. Purchase, H.C.: Which aesthetic has the greatest effect on human understanding? In: Di Battista, G. (ed.) GD 1997. LNCS, vol. 1353, pp. 248-261. Springer, Heidelberg (1997)

31. Purchase, H.C., McGill, M., Colpoys, L., Carrington, D.: Graph drawing aesthetics and the comprehension of uml class diagrams: an empirical study. In: APVis 2001: Proceedings of the 2001 Asia-Pacific symposium on Information visualisation, Darlinghurst, Australia, pp. 129-137. Australian Computer Society, Inc. (2001)

32. Recker, J., Dreiling, A.: Does it matter which process modelling language we teach or use? an experimental study on understanding process modelling languages without formal education. In: Toleman, M., Cater-Steel, A., Roberts, D. (eds.) 18th Australasian Conference on Information Systems, Toowoomba, Australia, pp. 356366. The University of Southern Queensland (2007)

33. Reijers, H.A., Mendling, J.: Modularity in process models: Review and effects. In: Dumas, M., Reichert, M., Shan, M.-C. (eds.) BPM 2008. LNCS, vol. 5240, pp. 20-35. Springer, Heidelberg (2008)

34. Russell, N., ter Hofstede, A.H.M., van der Aalst, W.M.P., Mulyar, N.: Workflow Control-Flow Patterns: A Revised View. BPM Center Report BPM-06-22, BPMcenter.org (2006)

35. Sarshar, K., Loos, P.: Comparing the control-flow of EPC and petri net from the end-user perspective. In: van der Aalst, W.M.P., Benatallah, B., Casati, F., Curbera, F. (eds.) BPM 2005. LNCS, vol. 3649, pp. 434-439. Springer, Heidelberg (2005)

36. TheFreeDictionary.com. Expertise (2009)

37. White, S.A.: Introduction to BPMN. BPTrends (July 2004)

38. zur Muehlen, M., Recker, J.: How much language is enough? theoretical and practical use of the business process modeling notation. In: Bellahsène, Z., Léonard, M. (eds.) CAiSE 2008. LNCS, vol. 5074, pp. 465-479. Springer, Heidelberg (2008) 